\title{
An Organophotocatalytic Borylative Minisci Reaction
}
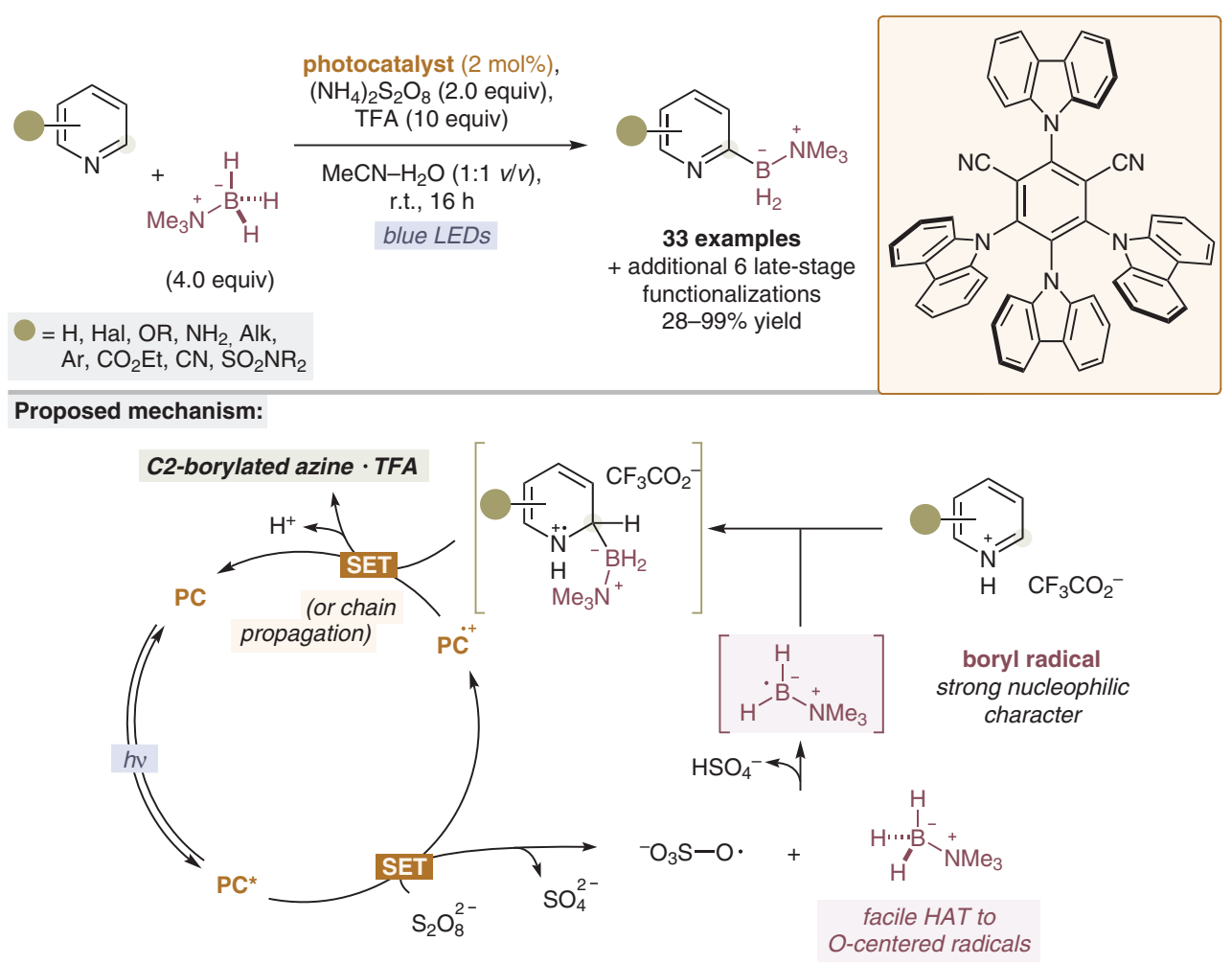

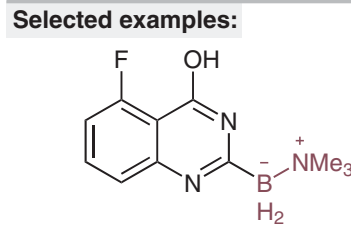

$91 \%$ yield<smiles>C[123I]c1ccc2ccccc2n1</smiles>

$63 \%$ yield $(\mathrm{C} 2 / \mathrm{C} 4=3: 1)$

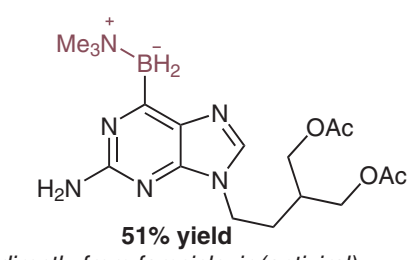

directly from famciclovir (antiviral)
Significance: Leonori and co-workers present an organophotocatalytic borylative Minisci reaction. Strongly acidic and oxidizing conditions ensure the generation of highly nucleophilic boryl radicals in situ, yielding C2-borylated nitrogen heterocycles with moderate regioselectivities and good to excellent yields. Additionally, the authors demonstrate that amine-borylated azines readily engage in conventional organoboron chemistry.
Comment: Polarity-reversal catalysis harnesses the electrophilic or nucleophilic character of radicals to render hydrogen atom-transfer reactions that would otherwise be polarity mismatched kinetically feasible. Because of the nucleophilic character of the respective radicals, amine-boranes themselves have previously been investigated as polarity-reversal catalysts (see Review below). The beauty of this work lies in the translation of this concept into one of the most sought-after transformations for the direct functionalization of N-heterocycles.

Review: B. P. Roberts Chem. Soc. Rev. 1999, 28, 25-35.
Key words

Minisci reaction

photocatalysis

azines

pyridines

quinolines

amine-borane

adduct 